\title{
Alteplase for the treatment of acute ischaemic stroke: NICE technology appraisal guidance
}

\section{H Chung, R R Camejo, D Barnett}

This NICE technology appraisal guidance considers the clinical and cost effectiveness of the use of alteplase for acute ischaemic stroke

\section{GUIDANCE}

This was published in the NICE technology appraisal guidance in June 2007. ${ }^{1}$

Alteplase is recommended for the treatment of acute ischaemic stroke when used by doctors trained and experienced in the management of acute stroke. It should only be administered in centres with facilities that enable it to be used in full accordance with its marketing authorisation.

\section{DEVELOPMENT OF THE GUIDANCE}

The Appraisal Committee recognised the high clinical and social burden associated with acute ischaemic stroke and understood the potential for these to be reduced through the thrombolytic action of alteplase. It was also conscious of the crucial role in successful stroke management of prompt assessment, supportive care and careful monitoring given in stroke units. While acknowledging the potential role of a thrombolytic treatment in resolving a stroke episode, the Appraisal Committee was also aware of its time-dependent efficacy and of the safety concerns around the use of alteplase, which mainly related to the incidence of potentially fatal intracranial haemorrhage.

The Appraisal Committee considered the evidence base for the clinical effectiveness of alteplase in acute ischaemic stroke from the five placebo-controlled, randomised clinical trials (NINDS I and II, ATLANTIS A and B and ECASS II) that assessed the efficacy of alteplase in this indication within its marketing authorisation. The Committee noted that the licensed dose of alteplase had to be given within 3 hours of onset of stroke symptoms after prior exclusion of symptomatic intracranial haemorrhage by appropriate imaging techniques. ${ }^{2-4}$ Meta-analysis of the trial data indicated that alteplase used in this way is associated with a statistically significant reduction in the risk of death or dependency at 3 months when compared with placebo. ${ }^{5}$ In addition, despite a statistically significant increase in the incidence of symptomatic intracranial haemorrhage in the alteplase arm in the first 7-10 days, the meta-analysis showed that there was no statistically significant difference between alteplase and placebo in all-cause mortality at 3 months.

The Appraisal Committee understood there were concerns about the National Institute of Neurological Disorders and Stroke (NINDS) Trials' protocols, which did not prevent a considerable imbalance in baseline stroke severity between treatment and control arms, and thus might influence the validity of conclusions drawn from these trials. Moreover, it appreciated that the metaanalysis results used in the appraisal were heavily influenced by these trial data. However, the Committee also took note of the discussion in an FDA review and in an independent study that further analysed the NINDS Trial data. ${ }^{67}$ It was aware of randomised controlled trial evidence of the use of alteplase beyond the 3-hour window and also took into account the results of a large pan-European observational study (SITS-MOST), which supported the conclusions drawn from the NINDS Trials.

An economic model was developed to estimate the lifetime cost effectiveness of alteplase in addition to best supportive care in comparison with best supportive care and medical management without thrombolytic agents. This showed that alteplase is more effective and less costly than the comparator and appeared robust to reasonable changes of the inputs used of costs or clinical effectiveness estimates. It further demonstrated that the probability that the incremental cost effectiveness ratio for alteplase is highly likely to be less than $£ 20000$ per quality-adjusted life year gained.

The Appraisal Committee discussed the relevance to the economic analysis of the cost of reorganising stroke services to enable the wide use of alteplase in accordance with its marketing authorisation, in particular the need for 24-hour access CT scanning and availability of doctors with specialist interest in acute stroke care. The Committee concluded that it would not be appropriate to include in its considerations the costs incurred in optimising services to enable the use of thrombolysis as it learnt that reorganisation of stroke services is already taking place through the National Stroke Strategy which include recommendations covering the set up of stroke centres with facilities suitable for treatment with alteplase.

The Appraisal Committee further considered the possibility that alteplase may be particularly effective and thus more cost effective when used in a specific subgroup of patients with acute stroke-for example, if administered in a shorter time frame. However, it considered that there was insufficient evidence from the trials to substantiate the subgroup analysis and therefore concluded that alteplase had not been clearly shown to be any more or less cost effective in particular subgroups of patients.

The Appraisal Committee concluded that the available evidence supported a positive recommendation of alteplase for the treatment of ischaemic stroke within its marketing authorisation. The evidence base indicated that the administration of alteplase together with the provision of best supportive care would not only be more clinically effective than best supportive care alone but might also result in long-term cost savings for the NHS. The Appraisal Committee emphasised, however, that alteplase should only be initiated by doctors trained and experienced in the management of acute stroke and in centres with facilities that enable it to be used in full accordance with its marketing authorisation.

The Appraisal Committee was aware that research is currently continuing to evaluate the safety and clinical effectiveness of alteplase beyond 3 hours after the onset of stroke symptoms. These studies, together with any potential studies in patients older than 80 years, would enable the assessment of the clinical and cost effectiveness of alteplase in these important groups, which represent a significant proportion of the patients who experience acute ischaemic stroke and are not currently included in the marketing authorisation. 


\section{Authors' affiliations}

H Chung, R R Camejo, D Barnett, National Institute for Health and Clinical Excellence, UK

Competing interests: None declared.

Correspondence to: Professor D Barnett, Department of Cardiovascular Sciences, Floor 4 RKCSB, Leicester Royal Infirmary, Leicester LE2 7LX, UK; dbbl@le.ac.uk

Accepted 28 August 2007

\section{REFERENCES}

1 National Institute for Health and Clinical Excellence (NICE). NICE technology appraisal guidance 122. Alteplase for the treatment of acute ischaemic stroke,
June 2007. Available at http://guidance.nice.org.uk/TA122/guidance/pdf/ English (accessed 22 August 2007).

2 The National Institute of Neurological Disorders and Stroke rt-PA Stroke Study Group. Tissue plasminogen activator for acute ischemic stroke. N Engl J Med 1995;333:1581-7.

3 Albers W, Clark M, Madden P, et al. ATLANTIS trial: results for patients treated within 3 hours of stroke onset. Alteplase Thrombolysis for Acute

Noninterventional Therapy in Ischemic Stroke. Stroke 2002;33:493-5.

4 Hacke W, Kaste M, Fieschi C, et al. Randomised double-blind placebo-controlled trial of thrombolytic therapy with intravenous alteplase in acute ischaemic stroke (ECASS II). Second European-Australasian Acute Stroke Study Investigators. Lancet 1998;352:1245-51.

5 Lloyd Jones $\mathrm{M}$, Holmes M. Alteplase for the treatment of acute ischaemic stroke: a single technology appraisal, February 2007.

6 Food and Drug Administration Clinical review for PLA 96-0350, June 1996. Available at http://www.fda.gov/cder/biologics/review/altegen061896r2.pdf (accessed 22 August 2007)

7 Ingall J, O'Fallon M, Asplund K, et al. Findings from the reanalysis of the NINDS tissue plasminogen activator for acute ischemic stroke treatment trial. Stroke 2004;35:2418-24.

\section{Commentary on NICE guidelines for alteplase for the treatment of acute ischaemic stroke}

\section{R I Lindley}

A nother important milestone for stroke has been reached with the recent approval by NICE of alteplase for acute ischaemic stroke. ${ }^{1}$ This welcome decision provides a much needed boost for those struggling to implement acute stroke intervention, for acute stroke intervention is certainly struggling. The sad truth about alteplase for acute ischaemic stroke is that treatment is still unavailable in a surprisingly large number of large hospitals in the UK and elsewhere.

The decision by NICE was based on a rigorous examination of the randomised controlled trial (RCT) data, and it is reassuring to note that this was based, not on the main positive trial, ${ }^{2}$ but on independent systematic reviews of the totality of the data. For those not familiar with these data, the main concern has been that most of the evidence of the effectiveness of alteplase within the 3-hour time window is based on one study- the National Institute of Neurological Disorders and Stroke (NINDS) study. ${ }^{2}$ However, concerns about this study have been examined in an independent reanalysis of the NINDS data and the main findings confirmed. ${ }^{3}$ The large postmarketing surveillance audit of alteplase in Europe, SITS-MOST, has also been reassuring: provided that alteplase is delivered by doctors trained and experienced in the management of acute stroke, results comparable to the RCT evidence can be achieved. ${ }^{4}$

Interestingly, the economic assessment of alteplase was in the context of treatment being delivered in well-organised stroke centres. It was considered unreasonable to factor in the large costs of stroke reorganisation in the economic modelling. This is reasonable given the impressive evidence that early brain imaging together with organised stroke unit care is the most important intervention in the management of acute stroke.

Implementation of NICE recommendations should follow as: "The Secretary of State has directed that the NHS provides funding and resources for medicines and treatments that have been recommended by NICE technology appraisals normally within 3 months...". ${ }^{1}$ However, this deceptively simple sentence hides enormous challenges. A comparison with the successful cardiological implementation of thrombolysis for acute myocardial infarction is instructive. Myocardial infarction is a painful frightening condition which prompts early calls for help and rapid hospital assessment, with coronary care units well established in the 1980s facilitating the completion of a series of coronary "mega-trials". The diagnosis of acute ST segment elevation myocardial infarction is relatively straightforward and the risks of cerebral haemorrhage low (3.9 per 1000 treated). ${ }^{5}$

In contrast, stroke is usually painless, and delays in attending hospital common. The diagnosis of stroke is often not straightforward and many doctors find neurological examination skills difficult, and the risk of symptomatic haemorrhagic transformation of infarction, or other intracranial haemorrhage, is high (at least 10-fold the risk compared with acute myocardial infarction). There is no easy equivalent to the ECG. Early CT scanning if often normal, early magnetic resonance scanning is not widely available, many older patients have contraindications to magnetic resonance, ${ }^{6}$ and diffusionweighted abnormalities can be absent even in definite stroke (and can be falsely positive due to non-stroke conditions). Stroke units are still not universally available. As a result of all these barriers, stroke thrombolysis rates in the UK and Australia are still well below $1 \%$. Rates are not much better elsewhere, with USA rates about $2 \% .^{7}$ Of course, there are exceptions to this appallingly low use, with many stroke centres achieving rates of $10-20 \%,{ }^{8}$ but these are the exception rather than the rule.

So, what has gone wrong with the implementation of stroke thrombolysis, some 12 years after the publication of the NINDS trial? First, the RCT evidence is inadequate. A mere 5727 patients contribute to the Cochrane Library review, whereas 58600 subjects were in the 1994 Fibrinolytic Therapy Trialists' overview. ${ }^{5}$ The evidence from the cardiological thrombolysis 